\title{
Interculturalité et apprentissage à distance en formation d'enseignants
}

Lamprini Chartofylaka, Elisabeth Odacre and Antoine Delcroix

\section{(2) OpenEdition}

Electronic version

URL: http://journals.openedition.org/ripes/2996

DOI: 10.4000/ripes.2996

ISSN: 2076-8427

\section{Publisher}

Association internationale de pédagogie universitaire

\section{Electronic reference}

Lamprini Chartofylaka, Elisabeth Odacre and Antoine Delcroix, "Interculturalité et apprentissage à distance en formation d'enseignants", Revue internationale de pédagogie de l'enseignement supérieur [Online], 37(1) | 2021, Online since 14 February 2021, connection on 16 February 2021. URL: http:// journals.openedition.org/ripes/2996 ; DOI: https://doi.org/10.4000/ripes.2996

This text was automatically generated on 16 February 2021.

Article L.111-1 du Code de la propriété intellectuelle. 


\title{
Interculturalité et apprentissage à distance en formation d'enseignants
}

\author{
Lamprini Chartofylaka, Elisabeth Odacre and Antoine Delcroix
}

\section{Introduction}

1 Le projet «Technologies éducatives pour l'enseignement en contexte» (TEEC) (Forissier et al., 2017) s'inscrit dans le cadre du développement des enseignements basés sur les effets de contextes (désormais EBEC) (Anjou, 2018; Forissier, 2019; Forissier et al., 2014). Le principe général de ces enseignements est de faire travailler des groupes d'apprenants appartenant à deux territoires distincts (en l'occurrence le Québec et la Guadeloupe) sur un même objet d'étude. Des outils numériques, notamment des échanges synchrones (en visioconférence) et asynchrones (grâce à un environnement numérique de travail), permettent les interactions entre apprenants portant sur les investigations que ceux-ci mènent sur chaque territoire. Un scénario pédagogique, s'appuyant notamment sur les différences (culturelles, sociétales, scientifiques...) entre les territoires concernés, est conçu de manière à favoriser l'émergence d'effets de contextes (Delcroix et al., 2013). Ces enseignements ont montré leur efficacité pour favoriser le développement de conceptions robustes chez les apprenants sur l'objet étudié (Anjou, 2018; Forissier et al., 2014).

2 Basé sur le principe du Design-Based Research (DBR) (Bourdeau, 2017), le projet TEEC intègre une démarche itérative faite de cycles d'analyse, de conception, de développement, d'évaluation et de révision. Nous rendons compte ici de la première des cinq itérations du projet, appelée "histoire et société ». Elle comportaient deux caractéristiques originales au sein de celui-ci : d'une part, il s'agissait de la seule phase du projet impliquant des étudiants de l'enseignement supérieur, les autres itérations concernant des élèves de l'école primaire et du début de l'enseignement secondaire; d'autre part, cette itération était la première expérimentation des EBEC dans le domaine des sciences humaines et sociales, alors que les travaux précédents les 
mettaient en œuvre dans le champ des sciences de la vie et de la terre (Anjou, 2018; Forissier et al., 2014).

3 En testant la transférabilité de la démarche des EBEC à des concepts de sciences humaines et sociales, l'itération « histoire et société » du projet TEEC a permis de mieux étudier l'importance des dimensions culturelles et sociales dans ce type d'enseignement, ce qui a pu être implémenté dans les itérations suivantes du projet.

4 Les étudiants impliqués dans l'étude dont nous rendons compte se destinent tous au métier d'enseignant (du primaire en Guadeloupe et du secondaire au Québec). Ainsi, nous cherchons à cerner si la participation à cette expérimentation a pu contribuer à la construction de compétences interculturelles (Byram, 2009; Dervin, 2004) chez ces futurs enseignants. Nous tentons également de déterminer si cette dimension interculturelle permet aux étudiants de rendre plus expertes leurs conceptions sur les concepts étudiés.

5 L'étude suit le plan suivant: nous commençons par présenter le cadre théorique retenu. Nous précisons ensuite la question de recherche, les hypothèses et la méthodologie choisie pour les instruire, basée sur une analyse des échanges synchrones entre les étudiants effectuée à partir des travaux de Byram (2009) et Dervin (2004). Nous poursuivons par la présentation des résultats, en portant particulièrement notre regard sur des extraits des corpus recueillis. Enfin, nous discutons ces résultats et concluons par quelques éléments relatifs aux particularités de cette expérimentation au sein du projet TEEC.

\section{Cadre théorique et hypothèses}

6 Cette partie commence par l'exposé du cadre dans lequel nous inscrivons les compétences interculturelles et sur la manière dont elles peuvent être appréhendées. Puis, nous présentons l'itération " histoire et société », en l'inscrivant simultanément dans l'économie générale du projet TEEC et dans ses dimensions particulières liées aux disciplines abordées et aux publics concernés. Enfin, nous précisons notre question de recherche, en relation avec d'autres travaux menés sur les EBEC.

\subsection{Coopération et apprentissage interculturel}

\subsubsection{De la culture et l'identité à l'apprentissage interculturel}

7 Plusieurs auteurs établissent un lien fort entre la culture, l'identité et le développement du sentiment d'appartenance à une collectivité. Ainsi, Rocher définit la culture comme « un ensemble lié de manières de penser, de sentir et d'agir plus ou moins formalisées qui, étant apprises et partagées par une pluralité de personnes, servent, d'une manière à la fois objective et symbolique, à constituer ces personnes en une collectivité particulière et distincte» (Rocher, 1969, p. 88). Guérin-Pace (2006) ajoute qu'un individu peut faire partie de plusieurs groupes d'appartenance (sociaux, religieux, familiaux, professionnels, etc.), mais la place qu'il accorde à chacun de ces groupes révèle son identité. Vinsonneau (2002) souligne le caractère dynamique des phénomènes liés à la culture et l'identité, provenant essentiellement des interactions sociales entre des personnes issues de groupes culturellement différents. 
8 Des travaux récents envisagent ces interactions dans une approche liée à la diversité culturelle et à l'interculturalité. Pour l'Organisation des Nations Unies pour l'Education, la Science et la Culture [UNESCO] (2005), la diversité culturelle concerne «la multiplicité des formes par lesquelles les cultures des groupes et des sociétés trouvent leur expression [...] quels que soient les moyens et les technologies utilisés. L'interculturalité « renvoie à l'existence et à l'interaction équitable de diverses cultures ainsi qu'à la possibilité de générer des expressions culturelles partagées par le dialogue et le respect mutuel». Ainsi, Pretceille (2015, p. 252) précise que «le passage de la différence à la diversité, du pluralisme à la pluralité, du multiculturalisme à l'interculturalité ne relève pas d'une simple évolution sémantique mais suggère un changement profond de perspective». Cette perspective oblige à "accepter de déconstruire le concept de culture au profit de l'interculturalité » (Pretceille, 2015, p. 251).

\subsubsection{Les compétences développées dans les approches interculturelles}

9 Certains auteurs distinguent les concepts de connaissances interculturelles et de compétences interculturelles. Ainsi, pour le Dictionnaire de l'éducation de qualité et de la culture de la paix (Graines de Paix, 2015), les connaissances interculturelles sont relatives aux «savoirs sur différentes cultures, tels que les lieux de vie, les traditions culinaires et vestimentaires, des notions sur d'autres langues, des aspects religieux, et des chansons, films et auteurs, c'est à dire des savoirs sur des choses assez tangibles ». Les compétences interculturelles se réfèrent aux attitudes, connaissances, facultés et actions (Huber et Reynolds, 2014) qui permettent d'engendrer « des comportements d'ouverture, d'accueil et d'écoute pour interagir avec d'autres cultures avec considération et aisance. » (Graines de Paix, 2015).

10 Les compétences interculturelles présentent donc des dimensions cognitives, affectives et comportementales, ainsi décrites par Dirks (1995, p. 84):

11 - la dimension cognitive fait référence à la capacité d'une personne de comprendre les similitudes et les différences entre sa propre culture et d'autres cultures, autrement dit fait état d'éléments relatifs à la conscience de soi et à la conscience culturelle;

12 - la dimension affective souligne l'aspiration d'une personne à reconnaître, apprécier et accepter les différentes cultures, autrement dit reprend des éléments relatifs à la sensitivité interculturelle (l'estime de soi, l'autosurveillance, l'empathie, l'ouverture d'esprit, le non-jugement et la relaxation sociale);

13 - la dimension comportementale comporte la capacité d'un individu d'atteindre les objectifs de la communication (flexibilité, gestion des interactions) quand il interagit avec les personnes d'une autre culture, autrement dit des éléments relatifs aux habilités interculturelles.

14 En résumé, les compétences interculturelles sont des capacités mobilisées chez un individu dans des situations où il est amené «à forger sa propre identité, à la fois singulière et multiple; à dépasser ses peurs et préjugés culturels; à reconnaître l'altérité, et à faire preuve d'ouverture, d'accueil, de compréhension, d'acceptation et d'inclusion.» (Graines de Paix, 2015). Il s'agit donc de situations où il prend note de «l'aptitude, dans la rencontre avec une personne d'une autre culture, de ressentir son propre enracinement sans subir de déracinement, sans craindre de perdre ses racines ou de les renier. » (Graines de Paix, 2015). 


\subsubsection{Le repérage et l'évaluation des compétences interculturelles}

Byram (1997) et Byram et al. (2002, p. 11-13) décrivent cinq catégories de ressources à mobiliser dans l'apprentissage des compétences interculturelles :

- une aptitude à la curiosité et à l'ouverture vers les autres qui permet de relativiser ses propres valeurs et croyances et réviser sa méfiance face à autrui (le « savoir-être »);

- des connaissances générales sur l'autrui (par exemple ses pratiques) et sur le fonctionnement et les valeurs des groupes sociaux (les « savoirs»);

- des compétences visant à interpréter correctement des sources et des évènements extérieurs et se rapportant à soi-même (le « savoir comprendre »);

- des compétences visant à découvrir et savoir mobiliser de nouvelles connaissances pendant une communication synchrone (le « savoir apprendre/faire »);

- une capacité à développer une conscience culturelle visant à évaluer de manière critique et objective sa propre culture et celle de son interlocuteur (le «savoir s'engager »).

Par la suite, Dervin (2004), soulignant l'importance de ce modèle et citant d'autres travaux comme ceux d'Abdallah-Pretceille (2003) et d'Abdallah-Pretceille et Porcher (1999), suggère qu'une expérience interculturelle fait appel aux principes suivants :

2 «a) Une ouverture à l'altérité (Abdallah-Pretceille et Porcher, 1999, p. 226) et le développement de capitaux interculturels;

b) Une connaissance de soi : "L'interrogation identitaire de soi par rapport à autrui fait partie intégrante de la démarche interculturelle" (Abdallah-Pretceille, 2003, p. 10);

c) Une négociation des rapports entre ses propres croyances, attitudes et significations et celles de l'Autre (Byram, 1997, p. 12), i.e. mettre fin à l'ethnocentrisme;

d) Une compétence d'interaction et d'analyse. Autrement dit, il s'agirait plus de "compréhension" que de "connaissances" sur l'Autre. »

Enfin, Byram (2009, p. 7), en reprenant ces éléments, précise que l'interculturalité permet le développement chez les individus de l'empathie et de la curiosité à l'égard des personnes issues d'autres cultures (point a), ainsi que d'une meilleure connaissance et compréhension de soi, afin d'évaluer ses propres modèles de pensées, de sentiments, d'émotions et de comportements (point b). Ainsi, les individus vont pouvoir interpréter et négocier des points de vue différents sans s'identifier à l'Autre ni adopter ses pratiques culturelles (point c) et entrer dans une perspective d'interaction et d'analyse afin de pouvoir agir ensemble dans des projets communs (point d).

27 Nos analyses relatives au développement de compétences interculturelles chez les étudiants, seront essentiellement basées sur les approches de Byram (2009) et Dervin (2004).

\subsubsection{Des situations d'apprentissage pour le développement des compétences interculturelles}

28 Pour les générations actuelles, entourées par le monde numérique, l'importance des compétences interculturelles est largement reconnue comme utile pour faciliter leur insertion professionnelle (Bartel-Radic, 2009). Par exemple, développer les compétences du $21^{\mathrm{e}}$ siècle (Lamri et al., 2018; Romero, 2016) peut être favorisé par la 
mise en œuvre de situations, dans des lieux physiques ou virtuels, incitant à la collaboration et à l'interaction entre les individus. Al Sahyouni Bou Fadel (2016) souligne que l'apprentissage au sein d'environnements numériques renforce les capacités d'un individu à agir, interagir et communiquer dans un contexte de travail collaboratif et interculturel. Dans ce cadre, les individus deviennent capables de construire et de verbaliser cette compétence en communiquant aux autres leurs visions des différences culturelles et en exprimant leurs émotions pendant ces situations de collaboration (Le Boterf, 2000) : elles leur permettent de comprendre ce que les autres expriment mais aussi de prendre conscience d'eux-mêmes en relation avec les autres (Henri et Lundgren-Cayrol, 2001, p. 61).

29 Ces dernières années, la mise en valeur de l'interculturalité en tant que compétence a été reconnue et mise en œuvre dans plusieurs dispositifs d'éducation formelle. L'intégration de la mobilité étudiante dans la formation universitaire (Onorati et D'Ovidio, 2016) ou des stratégies éducatives axées sur une éducation multiculturelle (Kvande et Lenz, 2013) en sont des exemples. Le projet TEEC comporte également cette dimension, par la mise en relation d'apprenants issus de deux pays différents, travaillant sur des mêmes objets et concepts, dans le cadre d'échanges médiatisés par ordinateurs.

\subsubsection{Les effets de contextes et le développement des compétences interculturelles}

30 Les effets de contextes ont été décrits par plusieurs auteurs (Delcroix et al., 2013; Merlo-Leurette et Forissier, 2009) comme la survenue au cours d'une situation d'apprentissage d'un décalage entre les conceptions des acteurs en présence, d'un côté celles de l'apprenant (liées à son propre contexte culturel ou scientifique) et de l'autre, celles véhiculées par les propositions d'enseignement. Ce phénomène peut en particulier survenir dans les pays où s'expriment plusieurs cultures alors que le système éducatif y demeure très centralisé, avec des programmes nationaux peu sensibles aux dimensions interculturelles, comme en France. Ces mêmes effets se produisent lorsque des apprenants vivant dans des territoires différents confrontent leurs conceptions (Forissier et al., 2014) sur un même objet d'investigation. C'est alors qu'une utilisation pédagogique de ces effets a été pensée, au travers du clash model (Forissier et al., 2013), mis en œuvre dans les EBEC (Anjou, 2018; Forissier, 2019) : un scénario pédagogique (portant sur l'acquisition d'un savoir ou d'une compétence) est pensé pour favoriser l'apparition de ces effets et mis en œuvre par deux classes appartenant à des territoires culturellement et physiquement différents. Le scénario s'appuie sur les écarts entre les caractéristiques de ces territoires relativement à l'objet étudié, ce qui provoque le clash lors des interactions. Des effets de contextes sont alors effectivement constatés et ont été décrits comme des séquences (Anjou, 2018), composées de trois phases : émergence de l'effet, développement de l'incompréhension entre apprenants puis grounding (Baker et al., 1999). Dans ce dernier moment, où se résout l'incompréhension, les trois dimensions des compétences interculturelles relevées par Dirks (1995) sont à l'œuvre: la dimension cognitive par la capacité à accéder au savoir de l'Autre; la dimension affective par l'acceptation de la culture d'autrui sans jugement et la dimension comportementale par l'expression d'une maitrise des interactions, dans l'objectif de comprendre des conceptions différentes exprimées sur le même objet. 


\subsection{L'itération « histoire et société » du projet TEEC}

Québec) et, sur chacun d'entre eux, la participation d'un enseignant d'histoire, d'enseignants-chercheurs, d'un doctorant et d'un référent technique. Elle est coordonnée par les concepteurs du projet TEEC qui s'assurent du respect des lignes directrices sur les plans pédagogiques et scientifiques. L'itération met en relation deux groupes d'étudiants se destinant au métier de professeur et bénéficiant tous d'un enseignement en didactique de l'histoire. Au Québec, les étudiants sont inscrits en deuxième année de formation en adaptation scolaire et sociale à l'Université du Québec à Montréal. Ce sont de futurs professeurs spécialistes de l'apprentissage et du comportement, qui exerceront le métier au niveau secondaire (selon l'organisation du système éducatif québécois), en particulier face à des élèves en difficultés. En Guadeloupe, les étudiants sont inscrits en première année de Master Métiers de l'enseignement de l'éducation et de la formation (MEEF) à l'École supérieure du professorat et de l'éducation de l'académie de Guadeloupe ${ }^{1}$, composante de l'Université des Antilles. Ce sont de futurs professeurs polyvalents, qui exerceront leur métier dans l'enseignement primaire (selon l'organisation du système éducatif français). Ainsi, les deux groupes d'apprenants suivent une formation les conduisant à enseigner plusieurs disciplines et ne possèdent pas, a priori, un intérêt particulier pour l'histoire.

Par ailleurs, le programme national français de l'école primaire (appliqué en Guadeloupe), et le programme de formation de l'école québécoise (Tableau 1) proposent un objectif commun, exprimé en des termes différents, qui est de comprendre en quoi les sociétés contemporaines sont héritières du passé. Cependant, le programme français, en ajoutant l'objectif d'une construction de « grands repères de l'histoire de France " (Ministère de l'Éducation nationale, 2018), semble posséder une dimension plus mémorielle, alors que le programme québécois insiste sur la préparation du futur citoyen à participer à « une société démocratique, pluraliste et ouverte sur un monde complexe » (Ministère de l'éducation, 2006, p. 337).

Tableau 1. Objectifs et compétences des programmes français et québécois

\begin{tabular}{|l|l|l|}
\hline & $\begin{array}{l}\text { Guadeloupe } \\
\text { Programme d'enseignement français au } \\
\text { cycle 3 } \\
\text { (Ministère de l'Éducation nationale, 2018) }\end{array}$ & $\begin{array}{l}\text { Québec } \\
\text { Programme de formation de l'école } \\
\text { québécoise au secondaire } \\
\text { (Ministère de l'éducation, 2006) }\end{array}$ \\
\hline Niveaux & Cycle 3 & Secondaire (1er cycle) \\
\hline Discipline & Histoire & Histoire et éducation à la citoyenneté \\
\hline
\end{tabular}




\begin{tabular}{|c|c|c|}
\hline Objectifs & $\begin{array}{l}\text { - Construire quelques premiers grands } \\
\text { repères de l'histoire de France } \\
\text { - Comprendre que le monde d'aujourd'hui } \\
\text { et la société contemporaine sont des } \\
\text { héritiers de longs processus, de ruptures, } \\
\text { de choix effectués par les femmes et les } \\
\text { hommes du passé }\end{array}$ & $\begin{array}{l}\text { Compréhension du présent à la lumière } \\
\text { du passé et le préparer à participer de } \\
\text { façon éclairée à la vie sociale, dans une } \\
\text { société démocratique, pluraliste et } \\
\text { ouverte sur un monde complexe }\end{array}$ \\
\hline Compétences & $\begin{array}{l}\text { - Situer chronologiquement des grandes } \\
\text { périodes historiques } \\
\text { - Ordonner des faits les uns par rapport } \\
\text { aux autres et les situer dans une époque } \\
\text { ou une période donnée } \\
\text { - Manipuler et réinvestir le repère } \\
\text { historique dans différents contextes } \\
\text { - Utiliser des documents donnant à voir } \\
\text { une représentation du temps et suscitant } \\
\text { la mise en perspective des faits } \\
\text { - Mémoriser les repères historiques liés } \\
\text { au programme et savoir les mobiliser } \\
\text { dans différents contextes }\end{array}$ & $\begin{array}{l}\text { - Interroger les réalités sociales dans } \\
\text { une perspective historique } \\
\text { - Interpréter une réalité sociale à l'aide } \\
\text { de la méthode historique } \\
\text { - Construire sa conscience citoyenne à } \\
\text { l'aide de l'histoire }\end{array}$ \\
\hline
\end{tabular}

Enfin, les compétences travaillées sont différentes. Le programme français insiste sur les compétences liées à la chronologie, à la mémorisation de repères historiques et leur mobilisation dans différents contextes, alors que le programme québécois se fonde sur le concept de réalités sociales, expression se rapportant à l'action humaine dans des sociétés d'hier ou d'aujourd'hui en y intégrant tous les aspects de la vie (culturel, économique, politique, territorial et social) (Ministère de l'éducation, 2006).

Ces différences d'approches ont rendu relativement complexe la préparation du scénario pédagogique. Cependant, des concepts communs ont pu être dégagés au-delà de la différence d'entrées constatée dans les programmes. Ainsi chaque cohorte a pu être scindée en trois groupes, chacun des groupes homologues investiguant des concepts communs (Tableau 2).

Tableau 2. Contenu disciplinaire de l'itération « histoire et société »

\begin{tabular}{|c|c|c|}
\hline & $\begin{array}{l}\text { Université des Antilles } \\
\text { (Guadeloupe, France) }\end{array}$ & $\begin{array}{l}\text { Université du Québec à Montréal (Québec, } \\
\text { Canada) }\end{array}$ \\
\hline \multirow{2}{*}{$\begin{array}{l}\text { Premiers } \\
\text { groupes }\end{array}$} & \multicolumn{2}{|c|}{ Concepts étudiés : pouvoir, monarchie, absolutisme } \\
\hline & Thème : le temps des rois & Réalité sociale : la christianisation de l'Occident \\
\hline \multirow{2}{*}{$\begin{array}{l}\text { Deuxièmes } \\
\text { groupes }\end{array}$} & \multicolumn{2}{|c|}{ Concepts étudiés : révolution, séparation des pouvoirs, régimes politiques } \\
\hline & $\begin{array}{l}\text { Thème: le temps de la } \\
\text { révolution et de l'empire }\end{array}$ & $\begin{array}{l}\text { Réalité sociale: les révolutions américaine ou } \\
\text { française }\end{array}$ \\
\hline
\end{tabular}




\begin{tabular}{|l|l|l|}
\hline \multirow{2}{*}{$\begin{array}{l}\text { Troisièmes } \\
\text { groupes }\end{array}$} & \multicolumn{2}{|l|}{ Concepts : machinisation/mécanisation, urbanisation, industrialisation } \\
\cline { 2 - 3 } & $\begin{array}{l}\text { Thème : l'âge industriel en } \\
\text { France }\end{array}$ & $\begin{array}{l}\text { Réalité sociale : l'industrialisation : } \\
\text { révolution économique et sociale }\end{array}$ \\
\hline
\end{tabular}

Le scénario pédagogique finalement retenu se résume ainsi : chaque groupe homologue doit se questionner sur les concepts retenus, écrire un scénario narratif en vue d'élaborer un tutoriel vidéo didactisé destiné à les expliquer. Des temps d'échanges synchrones entre équipes homologues ont pour objectifs de faire entrer les étudiants dans la collaboration, de les faire exposer leurs représentations sur les concepts investigués, puis de présenter les conclusions de leurs travaux et d'en dresser le bilan. Ces temps d'interactions synchrones sont espacés de quelques semaines pour permettre, dans l'intervalle, des moments de travail intra-groupe pour se questionner sur les concepts et effectuer des recherches documentaires.

\subsection{Question de recherche et hypothèses}

Ces dernières années, les travaux autour des EBEC se sont développés (pour une synthèse, voir : Forissier, 2019). Certains travaux (Anjou, 2018; Forissier et al., 2014) explorent leur contribution aux apprentissages et notamment la manière dont ils contribuent à la construction de conceptions expertes chez les apprenants. Par exemple, Anjou (2018) montre que des élèves du Québec et de la Guadeloupe comprennent que la géothermie s'appuie sur des échanges de chaleur et qu'il en existe plusieurs formes justifiant des usages différenciés en fonction des lieux. Ainsi la géothermie à haute énergie est utilisée en Guadeloupe pour produire de l'électricité, celle à basse énergie l'est au Québec pour réguler la température de locaux. Ces mêmes travaux et d'autres s'attachent à mieux décrire et comprendre le mécanisme des effets de contextes (Anjou, 2018; Le Bail, 2019) et démontrent le rôle des interactions synchrones entre apprenants dans leur développement et dans la résolution des malentendus d'ordre sociocognitifs qui s'y manifestent.

De manière complémentaire, ces travaux ont également contribué à faire émerger l'importance, dans ces effets, d'éléments relatifs à la méconnaissance de sa propre culture, de celle de l'Autre ou encore à la présence d'idées préconçues sur la culture ou les réalités sociales de l'Autre. Des phénomènes langagiers (Chartofylaka et al., 2018) en témoignent (par exemple: incompréhension d'un mot), ainsi que des changements d'états émotionnels au cours des interactions (Piot et al., 2019). La résolution de l'effet de contextes (grounding) semble s'effectuer au travers de la compréhension du contexte de l'Autre dans une approche plus générale (culturelle, linguistique, sociale...) que celle strictement liée aux objets étudiés. Ces constats nous ont incités à réaliser une analyse des échanges en visioconférence de l'itération " histoire et société » afin d'y repérer les éléments pouvant être mise en relation avec cette forme d'apprentissage interculturel. Nous formulons donc l'hypothèse principale suivante :

(H) : dans le cadre des EBEC, les échanges synchrones entre apprenants issus de contextes différents favorisent le développement de compétences interculturelles.

Comme les EBEC visent à développer des compétences plus expertes sur les sujets étudiés, nous tentons également de vérifier si ces échanges comportent des éléments 
liés aux conceptions des étudiants, relatives aux concepts étudiés, en tentant d'en cerner les évolutions au fil des séances de travail synchrone. Nous formulons l'hypothèse secondaire suivante :

(H') : dans le cadre des EBEC, les échanges synchrones favorisent le remaniement des conceptions des apprenants sur les concepts étudiés.

\section{Méthodologie}

41 Nous présentons la manière dont nous avons opéré le recueil de données et les dimensions éthiques ayant accompagnées ces expérimentations, avant de préciser les traitements réalisés.

\subsection{Déroulement et instrumentions des expérimentations}

Dans le respect de la planification des deux professeurs d'histoire, trois séances synchrones, d'une durée moyenne de 18 minutes, ont été programmées pour chaque groupe homologue. Sur les deux territoires, le dispositif de visioconférence a été isolé dans une salle située à proximité de la salle de cours principale. Les professeurs assuraient la gestion du groupe classe. Seuls les groupes d'étudiants participants aux expérimentations rejoignaient l'atelier de travail par visioconférence. Les chercheurs ont guidé ces étudiants et assuré l'enregistrement vidéo des échanges avec l'appui d'un référent technique. Une transcription automatique a d'abord été réalisée en utilisant NVivo $^{2}$. Ce premier matériel a été consolidé par des visionnages successifs des enregistrements vidéo, pour éliminer les erreurs de transcription, en prenant soin de noter les éléments non verbaux.

Les professeurs impliqués dans l'expérimentation ont proposé à leurs étudiants respectifs d'y participer, sur la base du volontariat, ce qui a permis de constituer les groupes homologues mentionnés ci-dessus. Les participants au projet (étudiants, professeurs, chercheurs...) ont renseigné et signé un formulaire de consentement de droit à l'image, établissant que les données récoltées seront exploitées uniquement à des fins de recherche, selon les règles éthiques du projet TEEC.

Dans cet article, nous rendons compte des interactions concernant les premiers groupes investiguant les concepts de monarchie, d'absolutisme et de pouvoir. Ces groupes ne comportent que des femmes, quatre en Guadeloupe et trois au Québec. Ces sept étudiantes, d'un âge moyen de 21 ans, n'ont pas suivi de formation spécifique en histoire au cours de leur scolarité.

\subsection{Méthode d'analyse des résultats}

Nous avons procédé à une analyse de contenu (Bardin, 2013) du matériel recueilli en commençant par une lecture flottante et chronologique pour en saisir le sens général (Savoie-Zajc, 2000). Ceci a permis d'isoler les énoncés qui témoignent d'éléments relatifs aux compétences interculturelles, repérés à l'aide des quatre principes décrits par Byram (2009) et Dervin (2004), ainsi synthétisés : (1) l'ouverture à l'altérité; (2) la connaissance de soi; (3) la négociation entre ses croyances propres et celles d'autrui; (4) la compétence d'interaction et d'analyse. L'ensemble de ces traitements a été réalisé en utilisant le package RQDA (Huang, 2016) du logiciel R ainsi qu'un tableur, ce qui a 
d'abord permis de vérifier la cohérence et l'exhaustivité du traitement opéré, avant d'établir des règles de numération (fréquence d'apparition) et de décrire le matériel. De manière plus précise, nous avons à la fois repéré des éléments de phrase qui traduisent l'expression d'un des principes ou qui montrent une compréhension réciproque et des "séquences d'interaction" qui traduisent la progression d'une compréhension réciproque. Ces dernières peuvent s'apparenter au phénomène de grounding observé dans les séquences d'effets de contextes (Baker et al., 1999). Des éléments non verbaux, associés à ces éléments évocateurs de compétences interculturelles, ont également été repérés. Il faut noter que certaines interactions peuvent renvoyer à plusieurs des principes, ce dont il a été tenu compte dans les décomptes présentés ci-après. Par exemple, l'interaction, reprise ci-dessous, illustre ce dernier point :

- Québec : Donc vous quand on vous nomme le sujet "la christianisation de l'Occident" vous pensez premièrement aux rois.

- Guadeloupe : Non nous on a le thème « Le temps de rois ». C'est à dire...

- Québec : Ok. Le temps des rois. Ok. Ah! [Étonnement] Donc le thème est différent mais je pense que certains sujets...vont se ramener à la même chose

- Guadeloupe : Exactement.

(Séance 1, 04 min 07 s) ${ }^{3}$

47 En effet, cette interaction témoigne, selon nous, à la fois d'un intérêt envers l'Autre et d'une capacité d'interaction et d'analyse.

\section{Résultats}

Comme nous l'avons indiqué plus haut, les quatre principes relatifs aux compétences interculturelles proposés par Byram (2009) et Dervin (2004) structurent la présentation des résultats.

\subsection{L'ouverture à l'altérité}

Nous avons repéré la présence d'éléments relatifs à l'ouverture à l'altérité dans 20 éléments ou interactions (Tableau 3), inégalement répartis entre les différentes séances.

Tableau 3. Fréquence d'apparition d'éléments relatifs à l'ouverture à l'altérité

\begin{tabular}{|l|l|l|l|}
\hline & Première séance & Deuxième séance & Troisième séance \\
\hline Fréquence d'apparition & 9 & 1 & 10 \\
\hline
\end{tabular}

Nous donnons maintenant deux exemples montrant comment s'opère dans les échanges cette ouverture à l'Autre.

51 Les phénomènes langagiers

52 Pour évoquer le même objet, la terminologie employée peut-être différente d'un territoire à l'autre, ce qui peut parfois provoquer une incompréhension initiale. Par exemple, pour nommer la production attendue (un tutoriel vidéo didactisé), les étudiants du Québec emploient le terme "baladodiffusion", proposé par l'office 
québécois de la langue française, terme usuel au Québec mais peu connu en Guadeloupe. Ceci provoque l'interaction suivante :

53

\section{[baladodiffusion]?}

- Guadeloupe : Dans la? [Étonnement]

- Québec : Dans la vidéo! [Rires]

(Première séance, $02 \mathrm{~min} 07 \mathrm{~s}-02 \mathrm{~min} 20 \mathrm{~s}$ ) provoque étonnement et curiosité : «Pourquoi vous avez choisi ce sujet-là? » (Québec, première séance, $03 \min 52 \mathrm{~s}$ ). Le groupe de Guadeloupe indique son intérêt pour la personne des rois : «c'était le plus passionnant. On aime bien les rois et tout ça du coup ça fait des personnalités quoi » (Guadeloupe, première séance, $03 \mathrm{~min} 55 \mathrm{~s}$ ). Des éléments non verbaux (silence, rires) témoignent de la surprise des membres du groupe québécois qui interprètent la réponse de leurs homologues et reformulent alors leur pensée "Donc vous quand vous nommez le sujet "la christianisation de l'Occident" vous pensez premièrement aux rois.» (Québec, première séance, 04 min $07 \mathrm{~s}$ ). Cette affirmation confirme l'intérêt manifeste des étudiantes pour comprendre la pensée de leurs homologues et provoque l'adhésion des étudiantes du Québec: «Ah! Donc le thème est différent mais [...] certains points vont se ramener à la même chose " (Québec, première séance, $04 \min 20 \mathrm{~s}$ ). Au fil des échanges, les deux groupes s'aperçoivent que, si les intitulés des entrées des programmes diffèrent, les concepts travaillés sont identiques, en accord avec les attentes de leur professeur respectif.

\subsection{La connaissance de soi et l'évaluation de ses propres modèles}

Nous avons repéré la présence d'éléments relatifs à cette compétence dans 18 échanges (Tableau 4), presque également répartis entre les différentes séances. 
Tableau 4. Fréquence d'apparition d'éléments relatifs à la connaissance de soi et à l'évaluation de ses propres modèles

\begin{tabular}{|l|l|l|l|}
\hline & Première séance & Deuxième séance & Troisième séance \\
\hline Fréquence d'apparition & 5 & 7 & 6 \\
\hline
\end{tabular}

Ces éléments relèvent principalement des sous-catégories suivantes dont nous illustrons la présentation par des extraits des échanges entre étudiants.

\section{La prise de conscience d'approches différentes pour l'enseignement de l'histoire}

Les interactions de la précédente séance, liées à l'entrée différente pour l'étude des mêmes concepts, a fait naitre le besoin d'approfondir le contenu des programmes de formation et des manuels du Québec pour y vérifier la présence du concept de royauté :

«Mais j'ai vraiment été sous le choc quand j'ai regardé tous mes manuels scolaires, ce n'était pas par rapport aux...rois, mais à la religion : Vraiment beaucoup la religion. On a parlé je pense d'un roi dans les manuels c'est tout! J'ai trouvé ça vraiment impressionnant de voir comment la même partie d'histoire peut être enseignée de manière différente. J'ai trouvé ça vraiment impressionnant mais à quel point aussi ça peut être plat nos manuels scolaires parce que c'est juste la religion. J'ai trouvé ça pénible à lire. Même les petits paragraphes, je les ai trouvés long. Mais ouais [...]» (Québec, deuxième séance, 00 min $35 \mathrm{~s}$ )

64 Le groupe québécois constate qu'il existe différentes approches pour l'enseignement de la même période historique. Cependant, les étudiantes s'accordent sur la pertinence de ces deux approches : «on peut y aller avec ces deux optiques vraiment différentes». (Québec, deuxième séance, 03 min $10 \mathrm{~s}$ )

La connaissance et l'évaluation du modèle didactique (programme, manuels)

Dans le premier tiers de la deuxième visioconférence, les étudiantes québécoises reviennent spontanément sur le contenu du projet de baladodiffusion :

67 «Mais c'est ça dans le fond le concept de monarchie je trouve qu'il est beaucoup plus exploité dans votre, dans votre thématique, parce que justement vous c'est le roi. Mais nous autres comme c'est la christianisation de l'Occident qui est dans le manuel on parle vraiment beaucoup de religion » (Québec, deuxième séance, 04 min $08 \mathrm{~s}$ )

Comme nous l'avons remarqué plus haut, les étudiantes du Québec se sont référées aux manuels pour approfondir leur connaissance de la réalité sociale servant de support à l'étude des concepts retenus. Elles sont également capables d'analyser ces manuels, voire de formuler des critiques : «j'ai trouvé cela pénible à lire » disait l'une d'entre elles dans un extrait cité plus haut. Elles renforcent cette impression négative en affirmant que «Pour vrai, je ne comprends pas comment on peut autant axer sur la religion, puis qu'on veut avoir le reste autour. C'est... Ça m'a vraiment découragé quand j'ai lu ça.» (Québec, deuxième séance, $09 \min 20$ s). Pour autant, l'idée d'un dépassement du modèle de référence (le programme de formation du Québec) existe : «Je suis comme « Ok... On peut dépasser le thème s'il vous plait? [Rires] » ». (Québec, deuxième séance, $05 \min 30 \mathrm{~s}$ ) 


\subsection{La négociation entre ses croyances propres et celles d'autrui} pouvoir. Quand on a vu monarchie, on a fait ok ce n'est pas juste la religion au final... [Rires] Donc c'est plus là qu'on se rejoint je pense...nous!

- Guadeloupe : Oui. [Accord avec hochement de la tête]

- Québec: Le concept monarchie, rejoint votre sujet des rois. Mais vous pouvez aussi sortir la religion dans votre sujet.

- Guadeloupe : Oui.

- Québec: Puisque que c'était le roi en ce moment-là qui avait le pouvoir. Je crois... [rires]. Je crois... Mais vous c'est quel roi?

- Guadeloupe : On en a quatre... Ah mais oui... Ce sont les rois qui représentaient la religion.

- Guadeloupe : Oui! Ils avaient un lien très fort avec la religion.

(Première séance, $05 \min 25 \mathrm{~s}-06 \mathrm{~min} 00 \mathrm{~s}$ )

73 Le groupe québécois semble ainsi convaincre le groupe homologue du lien entre la réalité sociale « christianisation » et le thème du programme en vigueur en Guadeloupe (« le temps des rois »).

74 Pourtant, cet accord reste fragile, comme en témoigne l'intervention, lors de la deuxième séance, d'une étudiante de Guadeloupe qui s'interroge : « Mais pourquoi elles parlent de religion?» (Guadeloupe, deuxième séance, $01 \min 29$ s). Une de ses coéquipières ajoute "nous ce n'est pas du tout axé sur la religion. Toujours pas... » (Guadeloupe, deuxième séance, 02 min $08 \mathrm{~s}$ ). Ainsi, au cours de cette séance, les étudiantes de Guadeloupe seront instigatrices d'un échange à front renversé en essayant de convaincre les étudiantes québécoises de la primauté du concept de royauté :

"- Québec : Pour réel, je ne comprends pas comment on peut autant axer sur la religion puis on peut avoir le reste autour. C'est... Ça m'a vraiment découragé quand j'ai lu ça. Ça me semble c'est plus intéressant de parler des rois là.

- Guadeloupe : Mais pourquoi vous ne faites pas la religion à travers le regard des rois? 
Ou un truc comme ça...

- Québec: On pourrait. Ça on pourrait. On pourrait faire c'est juste il faut trouver des sources quand-même valables. Je ne sais pas si...Je sais qu'on n'a rien trouvé pour le moment dans les livres mais... Parce qu'on trouve quand même il faut y aller avec le programme. Effectivement, c'est ça. Eh oui c'est l'angle qui est difficile à ramener. C'est vrai que c'est une très bonne idée. J'y pensais un peu mais... C'est ça, il faut trouver l'angle qui va être pertinent pour que ça rentre dans le programme. Dans le fond là, c'est une tâche plus complexe [rires].

- Guadeloupe : En fait, il vous faut trouver une bonne problématique qui le permettra.

(Deuxième séance, $09 \min 20 \mathrm{~s}-10 \mathrm{~min} 15 \mathrm{~s}$ )

La prise de conscience d'approches différentes dans l'enseignement des faits historiques, pousse les deux groupes d'apprenants à négocier l'arrimage de leurs idées pour nourrir le support numérique à produire. Cependant, si un accord se fait finalement autour d'une problématique liant les objets "religion » et "royauté », des limites interviennent dans ce processus, qui en souligne la difficulté : «Il n'est pas naturel de le comprendre [ce lien] quand on lit par exemple les manuels scolaires. » (Québec, deuxième séance, $04 \min 45 \mathrm{~s}$ ). D'une manière plus générale, les étudiantes québécoises mentionnent les exigences du programme de formation, comme étant un guide à respecter et qui fixe des limites à ne dépasser.

\subsection{La compétence d'interaction et d'analyse}

77 Nous avons repéré le développement de cette compétence dans 21 échanges (Tableau 6), répartis de manière quasiment égale entre les séances.

78 Tableau 6. Fréquence d'apparition d'éléments relatifs à la compétence d'interaction et d'analyse

\begin{tabular}{|l|l|l|l|}
\hline & Première séance & Deuxième séance & Troisième séance \\
\hline Fréquence d'apparition & 8 & 7 & 6 \\
\hline
\end{tabular}

79 Comme nous l'avons déjà indiqué, les étudiantes collaborent au fil des interactions pour arrimer leurs idées et développent ainsi leur capacité d'analyse des entrées des programmes et du contenu des manuels ce dont témoignent plusieurs échanges. Ainsi, le groupe guadeloupéen souligne que « [...] de toute façon la christianisation ça ne s'est pas, ça ne peut pas se faire sans le pouvoir des rois. Parce que c'est eux qui dirigent le pays donc...» (Guadeloupe, deuxième séance, $10 \mathrm{~min} 57 \mathrm{~s}$ ). Le groupe québécois exprime son accord avec ce point de vue : «Ouais. Ça c'est sûr. Il ne faut pas oublier ce point de vue-là. Souvent je trouve qu'ils oublient justement dans le manuel. » (Québec, deuxième séance, $11 \mathrm{~min} 09 \mathrm{~s}$ ).

De plus, les interactions conduisent les deux groupes à faire appel à des exemples prélevés dans leur contexte respectif, pour illustrer leur pensée :

81 «Tu sais par exemple, on a la croix sur le Mont Royal, on a des noms de rues qui ont des Saints [...] partout, jusqu'à il n'y a pas très longtemps on avait encore la croix sur les murs du Parlement » (Québec, troisième séance, $19 \min 20 \mathrm{~s}-20 \min 20 \mathrm{~s}$ ) 
82 On peut penser que l'étudiante concernée pense alors à la question de la séparation de l'église et de l'État au Québec, sujet d'actualité au moment de la réalisation de l'expérimentation. Dans une analyse assez symétrique, au sens où elle réfère au contexte historique et social de la Guadeloupe, une des étudiantes de ce territoire fait référence à son contexte historique, en affirmant que :

83 «il y aura toujours un lien avec la monarchie comment on peut dire hexagonale et notre propre histoire de colonisation. Mais c'est sûr qu'ici il n'y a pas de château ou bien on a des forts aussi [...] On voit l'empreinte des différents comment on appelle ça...Colonisateurs. » (Guadeloupe, troisième séance, $15 \mathrm{~min} 25 \mathrm{~s}$ )

$84 \mathrm{Au}$ fil des interactions, les deux groupes se rendent compte que leur regard sur les concepts étudiés est assez proche, ce qui leur semble être un bénéfice de leur participation à l'expérimentation :

85 « Mais c'est là que je comprends à quel point leur étude [TEEC] peut être intéressant justement parce que nos deux thèmes sont tellement abordés sur des branches différentes là. Puis, malgré cela on arrive quand même vers les mêmes définitions des concepts. C'est quand même fou! [Rires]» (Québec, troisième séance, 12 min $35 \mathrm{~s}$ )

86 Pourtant, l'analyse des échanges montre que les étudiantes des deux groupes conservent une conception différente de la production finale de leur vidéo :

87 - Québec : « Mais du coup ça va être vachement différent [nos vidéos] parce qu'au final on parle vraiment pas du tout de la...On ne parle pas de la même chose. »

- Guadeloupe : « Non c'est sûr ».

(Troisième séance, $12 \min 30 \mathrm{~s}-12 \min 34 \mathrm{~s}$ )

88 Ce point semble pouvoir être mis en relation avec les exigences ressenties par les étudiantes concernant les programmes respectifs. Cependant, les étudiantes québécoises estiment qu'il sera intéressant de s'interroger "peut-être dans un futur » FD lorsqu'elles seront professeures ${ }_{20}{ }_{20}$ de manière plus approfondie sur les liens existant entre les trois concepts et la société québécoise, afin de mieux appréhender le concept de monarchie.

\section{Discussion}

89 Nous présentons tout d'abord des éléments de discussion relatifs au développement des compétences interculturelles chez les étudiantes, avant de nous tourner vers la question du remaniement de leurs conceptions initiales sur les concepts historiques à étudier.

\subsection{L'expression et le développement de compétences interculturelles}

90 À notre sens, les résultats exposés plus haut montrent l'expression de compétences interculturelles chez les participantes à l'expérimentation. Pendant la première séance, les étudiantes font connaissance, ce qui provoquent des échanges relatifs à l'ouverture à l'Autre. Après le premier temps de travail réalisé de manière indépendante par chaque groupe, la deuxième séance voit des échanges plus marqués par la capacité à négocier entre ses croyances et celles de l'Autre, les étudiantes s'interrogeant sur la direction à donner à leur travail. Lors de la troisième séance, la production à remettre 
est quasiment achevée ou achevée et la forme de bilan que dressent les étudiantes de l'expérimentation les conduit à revenir sur ce que cette ouverture à l'Autre a pu leur apporter (10 éléments relatifs à cette catégorie). Sur un plan plus qualitatif, nous trouvons des indices du développement de compétences interculturelles dans la nature des échanges. Ceci s'exprime par l'attention portée à l'Autre. Par exemple, après avoir utilisé dans la première séance le terme "baladodiffusion », peu commun en Guadeloupe, les étudiantes du Québec vont éviter de le reprendre en lui substituant le terme plus commun de "vidéo ». De même une remarque (que nous n'avons pas reproduite) faite par le groupe de Guadeloupe sur l'accent des étudiantes québécoises disparait des échanges ultérieurs. Ces éléments peuvent être interprétés comme relevant des savoirs-être (Byram, 1997; Byram et al., 2002) mais s'inscrivent également dans la dimension affective (Dirks, 1995) des compétences interculturelles, en particulier dans leurs composantes d'autosurveillance et de non jugement.

De même, nous repérons plusieurs éléments relevant de la dimension cognitive des compétences interculturelles (Arasaratnam, 2016; Dirks, 1995). Par exemple, les interrogations suscitées par les entrées différentes des programmes provoquent une série d'échanges entre les deux groupes, mais aussi des actions hors temps d'échanges synchrones. Ainsi, les étudiantes du Québec vont s'assurer de l'exactitude de leurs souvenirs scolaires en se reportant aux manuels, qui leur semblent effectivement peu aborder les liens entre monarchie et religion (« mais à quel point aussi ça peut être plat nos manuels scolaires parce que c'est juste la religion ", Québec, deuxième séance, $01 \mathrm{~min} 05 \mathrm{~s}$ ). Ainsi, ces étudiantes expriment une capacité à comprendre les similitudes et les différences entre leur culture et celle de l'Autre. Le découragement initial, ressenti par les étudiantes, fait place à une forme d'enthousiasme à constater que les différences d'approches des phénomènes historiques dans les deux pays conduisent néanmoins à une définition analogue des concepts: "C'est quand même fou ", s'exclame une des étudiantes québécoises (troisième séance, 12 min $45 \mathrm{~s}$ ). De ce fait, les échanges semblent devenir moins formels dans la troisième séance synchrone. Chaque groupe exprime ainsi des liens entre plusieurs faits historiques ou sociétaux. Les étudiantes québécoises mentionnent la présence, jusqu'à une date récente, de la croix dans les tribunaux au Québec, symbolisant un lien entre le pouvoir et la religion. Les étudiantes de Guadeloupe semblent enfin oser exprimer un décalage entre l'histoire de la France et celle de la Guadeloupe, en évoquant la colonisation. Ainsi, le cœur des concepts étudiés par ces équipes est interrogé au travers de discours plus fluides, pouvant témoigner de l'expression de compétences comportementales (comme la prise de parole) dans la gestion des interactions.

93 Finalement, il semble que nous pouvons valider l'hypothèse $(\mathrm{H})$, puisque les trois séances successives témoignent d'un dépassement des points de vue initiaux, d'une réflexion sur les concepts s'effectuant lors des interactions, montrant ainsi un développement de compétences interculturelles. En demandant aux étudiantes d'investiguer un concept commun dans une alternance de phases de travail dans leur propre contexte et d'échanges synchrones en visioconférence, les EBEC créent une expérience interculturelle: dans le cadre de cette innovation pédagogique, un phénomène de traversée des frontières (Akkerman et Bakker, 2011) virtuel s'opère, permettant l'émergence de représentations sur les concepts historiques plus robustes, 
car mises à l'épreuve des différences socio-culturelles et historiques de leurs territoires respectifs.

94 Cependant, nous devons rester prudent quant aux conclusions tirées de cette expérimentation. En effet, la durée des échanges synchrones est relativement courte. Même si les analyses des échanges entre les deux autres paires d'équipes homologues, actuellement en cours, semblent conforter nos observations, nous nous questionnons sur la profondeur de ce développement de compétences, au-delà de l'effet immédiat venu de l'expérimentation elle-même. Un point rassurant peut résider dans l'expression par une des étudiantes québécoises d'un possible retour sur ce qu'elle a appris lors de l'expérimentation dans sa future activité de professeure.

Enfin, les étudiantes québécoises se sont davantage engagées dans les interactions que leurs homologues de Guadeloupe. Nous ne pouvons trancher entre l'existence de facteurs intra personnels ou plus contextuels : les étudiantes de Guadeloupe étaient, au moment de l'expérimentation, en phase de préparation au concours de recrutement de professeur des écoles, concours dont les épreuves écrites sont centrées sur le français et les mathématiques. De ce fait, elles étaient peut-être moins motivées par le thème « histoire et société ».

\subsection{Le remaniement des conceptions initiales des apprenants} absolutisme s'est effectué, par les enseignants en charge des classes concernées, au travers du thème «le temps des rois" en Guadeloupe et de la réalité sociale «la christianisation de l'occident » au Québec. L'absence de lien apparent entre ces entrées provoque d'emblée chez ces étudiantes sans formation spécifique en histoire une interrogation dont nous avons rendu compte (« pourquoi encore la religion? », Québec, première séance, $03 \mathrm{~min} 18 \mathrm{~s}$ ). Les conceptions initiales des étudiantes semblent fortement liées au curriculum de chacun des pays, centré sur la question de la christianisation au Québec et laissant une large place à la monarchie en Guadeloupe (" On aime bien les rois et tout ça », Guadeloupe, première séance, 03 min 55 s).

Ce n'est que d'une façon progressive et non linéaire que ces conceptions initiales vont être réexaminées. Ainsi, si dès la première séance synchrone, les étudiantes s'interrogent sur la relation entre les concepts à étudier et les entrées qui leur sont proposées, le questionnement resurgit au début de la deuxième séance («Mais pourquoi elles parlent de religion? », Guadeloupe, deuxième séance, $01 \min 29$ s). Ce n'est que lors de la troisième séance que le lien entre les concepts étudiés et chacune des entrées des programmes est plus fortement établi, montrant la compréhension des relations étroites entre monarchie et religion dans l'Occident ( Puis, malgré cela on arrive quand même vers les mêmes définitions des concepts", Québec, troisième séance, $12 \mathrm{~min} 35 \mathrm{~s}$ ). Ainsi, les étudiantes surmontent l'effet de contextes provoqué par les entrées thématiques différentes et conçoivent que ces entrées peuvent conduire à interroger les mêmes concepts. Ce phénomène de grounding (Baker et al., 1999), témoignant de l'expression de compétences interculturelles (Dirks, 1995), s'accompagne de changements d'états émotionnel chez les étudiantes (par exemple marqués par des rires). Il participe au remaniement des conceptions des apprenants (Anjou, 2018) comme nous l'avons constaté au travers du discours des étudiantes, notamment lors de la troisième séance, avec un engagement plus fort dans la 
discussion sur les concepts et leur exemplification au travers d'éléments prélevés dans le contexte propre à chacun des pays.

Nous restons cependant prudents sur la validation totale de l'hypothèse secondaire (H'). En effet, l'analyse des interactions montre bien un changement de conception, par l'acceptation d'approches différentes sur les mêmes concepts historiques. Cependant, les étudiantes restent persuadées que leurs productions finales seront néanmoins conceptuellement différentes (" On ne parle pas de la même chose », Québec, troisième séance, 12 min $32 \mathrm{~s}$; « Non, c'est sûr ", Guadeloupe, troisième séance, 12 min 34 s). Nous pouvons penser que plusieurs facteurs explicatifs se combinent ici. Par exemple, l'importance accordée par les étudiantes aux programmes officiels de leur pays respectifs et la croyance que les manuels les traduisent fidèlement (Karwera, 2012) peuvent les conduire à maintenir l'entrée par les thèmes ou réalités sociales (selon le pays), plutôt qu'à se focaliser sur les concepts étudiés. Les consignes données par les enseignants au début de l'expérimentation peuvent aussi constituer un élément explicatif, tout comme l'évaluation finale du diplôme préparé (Québec et Guadeloupe) ou le concours de recrutement (Guadeloupe). Ces derniers éléments sont contextuellement ancrés (Odacre, 2018) et peuvent être vus comme prédominants par les étudiantes, puisqu'ils conditionnent leur avenir professionnel.

\section{Conclusion}

Nous avons exposé, dans cet article, les résultats relatifs aux échanges entre les premiers groupes homologues de l'itération "histoire et société » du projet de recherche TEEC. La tâche assignée à ces deux groupes d'apprenants était la production d'une capsule vidéo didactisée, portant sur les concepts de pouvoir, monarchie, absolutisme, au travers des entrées des programmes «le temps des rois» en Guadeloupe et "la christianisation de l'occident» au Québec. Les entrées différentes retenues pour aborder les concepts à étudier ont favorisé l'émergence d'effets de contextes (Delcroix et al., 2013). Le dépassement de l'obstacle à la base de ces effets a été vecteur de développement de compétences interculturelles et de remaniement des conceptions sur les concepts étudiés chez les participantes.

Par ailleurs, l'expérimentation "histoire et société » présentait deux caractéristiques singulières au sein du projet TEEC : d'une part, les apprenants concernés relevaient de l'enseignement supérieur et, d'autre part, il s'agissait de la première expérimentation des EBEC en sciences humaines et sociales. Sur le premier point, nous avons constaté l'existence de plusieurs contraintes, sans doute supérieures à celles constatées dans l'enseignement primaire et secondaire, liées à l'organisation des diplômes concernés par l'expérimentation et à leur finalité. L'engagement des étudiantes ayant participé à l'expérimentation a pu être freiné par les craintes qu'elles nourrissaient quant à l'obtention du diplôme (Québec et Guadeloupe) ou la réussite au concours (Guadeloupe). Sur ce plan, nous rendrons compte dans un article ultérieur du point de vue des professeurs qui ont participé à l'expérimentation, mais nous pouvons mentionner, dès à présent, qu'ils ont le même ressenti que les étudiantes. À cela s'ajoute au Québec, la question de l'évaluation par les étudiants des enseignements ce qui ne permet pas, selon l'enseignant québécois, de s'écarter du programme d'étude. Sur le second point, le concept d'EBEC, initialement expérimenté pour des enseignements en sciences de la vie et de la terre (Forissier et al., 2014), a fonctionné 
dans l'itération « histoire et société » en produisant des effets de contextes (Delcroix et al., 2013) qui ont contribué, comme nous l'avons dit, à nourrir les compétences interculturelles des étudiants. Par ailleurs, l'itération «conte» de ce même projet a montré la capacité des EBEC à participer au développement des compétences langagières d'élèves du primaire (Chartofylaka et al., 2018). Ce type d'enseignement semble donc être pertinent à tous les niveaux d'enseignement et dans une large variété de champs disciplinaires.

101 Ainsi, en prenant les précautions nécessaires pour les inscrire dans les programmes d'étude et dans les objectifs que se fixent les apprenants concernés, les EBEC semblent pouvoir constituer une des réponses pour le développement des compétences $\mathrm{du} 21^{\mathrm{e}}$ siècle (Lamri et al., 2018; Romero, 2016) par la collaboration et l'interaction entre les individus qu'ils mettent en œuvre.

\section{Remerciements}

Les travaux présentés dans cet article ont été financés par le projet TEEC (ANR-FRQSC no 2017-QF-210862). Les auteurs remercient les enseignants qui ont participé à l'itération sociohistoire, Danny Legault (Québec) et Valérie Hugues (Guadeloupe) et les membres du projet TEEC qui l'ont accompagnée, Diane-Gabrielle Tremblay, Geneviève Demers, Frédéric Fournier (Québec), Marcel Héréson (Guadeloupe), ainsi que les étudiants des deux territoires.

\section{BIBLIOGRAPHY}

Abdallah-Pretceille, M. (2003). Former et éduquer en contexte hétérogène : pour un humanisme du divers. Economica/Anthropos.

Abdallah-Pretceille, M. et Porcher, L. (1999). Diagonales de la communication interculturelle. Economica/Anthropos.

Akkerman, S. F. et Bakker, A. (2011). Boundary crossing and boundary objects. Review of Educational Research, 81(2), 132-169. https://doi.org/10.3102/0034654311404435

Al Sahyouni Bou Fadel, R. (2016). L'interopérabilité culturelle et l'interculturalité : le modèle de Vifralie. Communication, 34(1), 1-17. https://doi.org/10.4000/communication.6636

Anjou, C. (2018). L'énergie géothermique : représentation, contextes et enseignements aux Antilles et dans la zone Caraibe [thèse de doctorat, Université des Antilles, France]. Thèses.fr. http:// www.theses.fr/2018ANTI0318

Arasaratnam, L. A. (2016). Intercultural Competence. Oxford Research Encyclopedia of Communication, 1-20. https://doi.org/10.1093/acrefore/9780190228613.013.68

Baker, M., Hansen, T., Joiner, R. et Traum, D. (1999). The role of grounding in collaborative learning tasks. Dans P. Dillenbourg (dir.), Collaborative Learning: Cognitive and Computational Approaches. Elsevier Science. 
Bardin, L. (2013). L'analyse de contenu. Presses Universitaires de France.

Bartel-Radic, A. (2009). La compétence interculturelle : état de l'art et perspectives. Management international, 13(4), 11-26. https://doi.org/10.7202/038582ar

Bourdeau, J. (2017). The DBR methodology for the study of context in learning. Dans P. Brézillon, R. Turner et C. Penco (dir.), Modeling and Using Context (vol. 10257, p. 541-553). Springer International Publishing. https://doi.org/10.1007/978-3-319-57837-8_44

Byram, M. (1997). Teaching and assessing intercultural communicative competence. Multilingual Matters.

Byram, M. (2009). Sociétés multiculturelles et individus pluriculturels : le projet de l'éducation interculturelle. Conseil de l'Europe. https://rm.coe.int/CoERMPublicCommonSearchServices/ DisplayDCTMContent?documentId=09000016805a223d

Byram, M., Gribkova, B. et Starkey, H. (2002). Developing the intercultural dimension in language teaching: A practical introduction for teachers. Conseil de l'Europe. https://rm.coe.int/16802fc1c3 Chartofylaka, L, Anciaux, F., Candau, O.-S., Jeannot-Fourcaud, B., Carignan, I. et Saint-Pierre, A. (2018). Il était une fois des contes en contextes : retour sur une expérimentation au primaire en Guadeloupe et au Québec. Contextes et didactiques, 11(1), 67-108. https://doi.org/10.4000/ced.1014

Delcroix, A., Forissier, T. et Anciaux, F. (2013). Vers un cadre d'analyse opérationnel des phénomènes de contextualisation didactique. Dans F. Anciaux, T. Forissier et L. F. Prudent (dir.), Contextualisations didactiques, approches théoriques (p. 141-185). L'Harmattan.

Dervin, F. (2004). Définition et évaluation de la compétence interculturelle en contexte de mobilité : ouvertures. Moderna språk, 98(1), 68-77. https://users.utu.fi/freder/mob.pdf

Dirks, D. (1995). The quest for organizational competence: Japanese management abroad. Dans B. N. Kumar (dir.), Management and International Review (p. 75-90). Gabler Verlag. https://doi.org/ 10.1007/978-3-322-90985-5_6

Forissier, T. (2019). Contextualisation et effets de contextes dans l'apprentissage des sciences [Habilitation à diriger des recherches, Université des Antilles, France]. HAL. https://hal.archivesouvertes.fr/tel-02277162

Forissier, T., Bourdeau, J. et Fécil, S. (2014, octobre). Interfaces élève-machine pour apprendre à partir des contextes [communication par affiche]. IHM'14, $26^{\mathrm{e}}$ conférence francophone sur l'Interaction Homme-Machine. Lille, France. https://hal.archives-ouvertes.fr/hal-01089623

Forissier, T., Bourdeau, J., Mazabraud, Y. et Nkambou, R. (2013). Modeling context effects in science learning: The clash model. Dans P. Brézillon, P. Blackburn et R. Dapoigny (dir.), Modeling and Using Context (vol. 8175, p. 330-335). Springer. https://doi.org/10.1007/978-3-642-40972-1_25

Forissier, T., Bourdeau, J. et Psyché, V. (2017). Quand les contextes se comparent et se parlent. Contextes et didactiques, 10. https://doi.org/10.4000/ced.954

Guérin-Pace, F. (2006). Sentiment d'appartenance et territoires identitaires. Espace géographique, 35(4), 298-308. https://doi.org/10.3917/eg.354.0298

Henri, F. et Lundgren-Cayrol, K. (2001). Apprentissage collaboratif à distance : pour comprendre et concevoir les environnements d'apprentissage virtuels. Presses de l'Université du Québec.

Huang, R. (2016). RQDA: R-based qualitative data analysis. R package version 0.2-8. http://rqda.rforge.r-project.org/

Huber, J. et Reynolds, C. (2014). Les composantes de la compétence interculturelle. Dans J. Huber et C. Reynolds (dir.), Développer la compétence interculturelle par l'éducation (p. 83-86). Conseil de 
l'Europe. https://www.cairn.info/developper-la-competence-interculturelle--9789287177452p-83.htm

Karwera, V. (2012). La transposition didactique du concept de citoyenneté à travers des pratiques d'enseignement de l'histoire au secondaire [thèse de doctorat, Université du Québec à Chicoutimi et Université du Québec à Montréal, Canada]. Archipel. https://archipel.uqam.ca/4987/1/D2318.pdf

Kvande, L. et Lenz, C. (2013). Pratique de l'apprentissage interculturel. L'exploration de concepts par de futurs enseignants norvégiens. Revue internationale d'éducation de Sèvres, 63, 111-121.

https://doi.org/10.4000/ries.3495

Lamri, J., Barabel, M., Meier, O., Burger, P. et Lesaffre, J. (2018). Les compétences du $21^{e}$ siècle. Dunod.

Le Bail, C. (2019). Apprentissage basé sur la confrontation à distance de contextes. Analyse des interactions verbales, caractérisation des effets de contexte et recommandations [Rapport de recherche postdoctorale]. Paris Télécom et Université Paris V Descartes.

Le Boterf, G. (2000). Construire les compétences individuelles et collectives. Éditions d'Organisation.

Merlo-Leurette, S. et Forissier, T. (2009). La contextualisation dans l'enseignement des sciences et techniques en Guadeloupe. Grand N, 83, 19-26.

Ministère de l'éducation nationale. (2018). Programme d'enseignement français au cycle 3. Gouvernement de France. https://cache.media.eduscol.education.fr/file/ programmes_2018/20/2/Cycle_3_programme_consolide_1038202.pdf

Ministère de l'éducation. (2006). Programme d'Histoire et éducation à la citoyenneté. Programme de formation de l'école québécoise au secondaire. Gouvernement du Québec. http:// www.education.gouv.qc.ca/fileadmin/site_web/documents/education/jeunes/pfeq/ PFEQ_histoire-education-citoyennete-premier-cycle.pdf

Odacre, E. (2018). L'enseignement des sciences économiques et gestion en BTS en Guadeloupe : entre prescrits nationaux et contexte socio-économique régional [thèse de doctorat, Université des Antilles, France]. Thèses.fr. https://www.theses.fr/2018ANTI0252

Graines de Paix. (2015). Dictionnaire de l'éducation de qualité et de la culture de la paix. https:// www.grainesdepaix.org/fr/ressources-de-paix/dictionnaire-education-paix

Onorati, M. et D’Ovidio, F. (2016). Mobilité et compétence interculturelle dans les programmes internationaux d'apprentissage tout au long de la vie : une étude longitudinale. Journal of International Mobility, 4(1), 109-128. https://doi.org/10.3917/jim.004.0109

Organisation des Nations Unies pour l'éducation, la science et la culture (UNESCO). (2005). Convention sur la protection et la promotion de la diversité des expressions culturelles. https:// unesdoc.unesco.org/ark:/48223/pf0000142919_fre

Piot, M., Alabarbe, T., Goanzalez, J., Le Bail, C., Prevost, L., Bourdeau, J., Bernard, F. X., Baker, M. et Détienne, F. (2019). Joint analysis of verbal and nonverbal interactions in collaborative e-learning. $8^{\text {th }}$ International Conference on Affective Computing and Intelligent Interaction Workshops and Demos (ACIIW). IEEE. https://doi.org/10.1109/ACIIW.2019.8925033

Pretceille, M. (2015). L'interculturel comme paradigme de transgression par rapport au culturalisme. Voix Plurielles, 12(2), 251-263. https://doi.org/10.26522/vp.v12i2.1286

Rocher, G. (1969). Introduction à la sociologie générale. Éditions Hurtubise. 
Romero, M. (2016, 13 février). Compétences pour le $21^{e}$ siècle. https://margaridaromero.me/ 2016/02/13/competences-du-21e-siecle/

Savoie-Zajc, L. (2000). L'analyse de données qualitatives : pratiques traditionnelle et assistée par le logiciel NUD•IST. Recherches qualitatives, 21, 99-123.

Vinsonneau, G. (2002). Le développement des notions de culture et d'identité : un itinéraire ambigu. Carrefours de l'éducation, 14(2), 2-20. https://doi.org/10.3917/cdle.014.0002

\section{NOTES}

1. Devenue en septembre 2019 Institut supérieur du professorat et de l'éducation de l'académie de la Guadeloupe.

2. Logiciel d'analyse qualitative édité par QSR International. Voir: https:// www.qsrinternational.com/nvivo-qualitative-data-analysis-software/home.

3. Ce codage, employé dans la suite de l'article, indique le début de l'échange retranscrit.

\section{ABSTRACTS}

This study reports on an experiment, carried out within the framework of the "Educational technologies for teaching in context - TEEC" project, putting in collaboration students from the Université des Antilles with students of the Université du Québec à Montréal. These students undertake degree-level studies to become future teachers and both groups of students benefit from a course in history education. The common and specific cultural characteristics of the two territories were used in an educational scenario whose objective was the construction of rigid conceptions on historical social concepts - common in the two training curriculums. A series of synchronous communication exchanges (via videoconferencing) between students shows that they become aware that the same concepts can be taught through different thematic entries, depending on the specific socio-cultural elements of each territory. However, their conceptions remain to a great extent conditioned by the approaches used in the school education program they pursued.

L'étude rend compte d'une expérimentation, réalisée dans le cadre du projet «Technologies éducatives pour l'enseignement en contexte - TEEC ", ayant mis en contact des étudiants de l'Université des Antilles et de l'Université du Québec à Montréal. Ces étudiants se destinent au métier de professeur et bénéficient tous d'un enseignement d'histoire. Les traits culturels (communs et spécifiques) des deux territoires ont été exploités dans un scénario pédagogique élaboré avec l'objectif de contribuer à la construction de conceptions robustes sur des concepts sociaux historiques communs aux deux programmes de formation. Une série d'échanges synchrones (par visioconférence) entre les étudiants montre qu'ils prennent conscience que les mêmes concepts peuvent être enseignés au travers d'entrées thématiques différentes, dépendant d'éléments socioculturels propres à chaque territoire. Cependant, leurs conceptions restent conditionnées par les approches retenues dans l'enseignement scolaire qu'ils ont suivi. 
INDEX

Mots-clés: Technologies éducatives, travail collaboratif, diversité culturelle, effets de contextes, conceptions

\section{AUTHORS}

\section{LAMPRINI CHARTOFYLAKA}

Centre de Recherches et de Ressources en Éducation et Formation, Abymes, Guadeloupe, lamprini.chartofylaka@gmail.com

\section{ELISABETH ODACRE}

Centre de Recherches et de Ressources en Éducation et Formation, Abymes, Guadeloupe, elisabeth.odacre@gmail.com

\section{ANTOINE DELCROIX}

Centre de recherches et de ressources en éducation et formation, Abymes, Guadeloupe, antoine.delcroix@univ-antilles.fr 